\title{
Investigation of parts assembly error, taking into account the deviation of the shape of their surfaces.
}

\author{
Mikhail Bolotov ${ }^{1, *}$, Iliya Grachev ${ }^{1}$, and Evgeny Kudashov ${ }^{1}$ \\ ${ }^{1}$ Samara University, Institute of Engines and Power Plants, 443086 Samara, Russian Federation
}

\begin{abstract}
In this article, we study the errors in the assembly of parts, taking into account the deviation of the shape of their surfaces. The developed computer model of the assembly of the engine low-pressure turbine rotor is designed to predict the values of the assembly parameters, such as radial and face run-out. The forecasting of the above assembly parameters is carried out based on the data of actual dimensions and shape of the surfaces of parts assembled as an assembly unit. The analysis of study results made it possible to obtain a conclusion about the qualitative influence of geometrical errors of the assembled parts on the error of the assembly parameters.
\end{abstract}

\section{Introduction}

The assembly of products is an important stage in their production. The problems of manufactured products quality improvement, cost reduction of their production and time decrease for serial production preparation are particularly important. The solution of these problems can be achieved by developing and applying modern technological processes that widely use digital technologies for predictive modeling and control. Digital technologies can provide the walkthrough, traceability of quality indicators, rational solution of accuracy issues based on predictive modeling data. In the area of study, there are works that suggest the use of measurement results to ensure the accuracy of production [1, 2]. The development of reliable digital models that allow to predict product quality parameters applicable in manufacturing conditions is a difficult task. Digital models must take into account a complex nature of the surface shape deviation and the stress-strain state of the products.

The skin model [3] is a measurable surface, which must be parameterized (filtered) in order to take into account errors. There are published works that include studies of shape deviation effect on the accuracy of parts mating $[4,5]$.

In the article [6], a method is proposed for taking into account surface shape and deformation variations while predicting the accuracy of assembly parameters. The forecasting the stress-strain state in the proposed method can be performed using CAE systems.

\footnotetext{
*Corresponding author: grachmalek2602@gmail.com
} 
In the process of models development, it is necessary to implement a set of measures including verification and refinement of structure and parameters. The paper considers the problem of studying of parts assembly errors, taking into account the deviation of the shape of their surfaces using the developed predictive models. The simulators of disk and spacer of the low-pressure turbine as taken as parts. The mating of turbine parts should meet high requirements for accuracy of radial and face run-outs affecting the irregularity of the radial clearance between the periphery of the blades and the inner surface of the stator. The value of the radial clearance and its irregularity exerts a direct influence on the efficiency of the engine [7].

The methods for predicting the accuracy of assembly parameters can be used to supplement and expand the capabilities of parametric models of machine tooling [8].

\section{Modeling the parts assembly}

It is known that the accuracy of parts assembly is affected by a variety of factors, including: the deviation of the shape and location of the mating surfaces and their contact deformations, volume strain of the parts, irregular application of the tightening force of bolt joints and other factors. In this article, we will consider the influence of the deviation of the shape of the interfaced surfaces on the accuracy of assembly parameters using a technique that takes into account the real geometry of parts surfaces. Since the assembly of parts features a complex stress-strain state, a complex shape deviation and a contact interaction of the mating surfaces, CAD CAE systems are used to predict the interfaced state.

\subsection{Creating valid 3D models}

The creation of valid parts models was carried out in the following sequence. At the first stage, the building of nominal models of mating parts was performed. At the second stage, the actual (model) mating surfaces were calculated by the finite set of points based on nominal equations, form deviation functions and location deviation parameters. At the third stage, the nominal models were rebuilt by adding real (model) surfaces to them, so that they bound the contour of the part and create a unique closed body.

The deviation of the shape of parts mating surfaces was represented by a function of the harmonic series:

$$
\delta_{n}(x)=\sum_{k=1}^{+\infty} A_{k} \sin \left(k \cdot\left(2 \cdot \pi \cdot x / \tau+\theta_{k}\right)+\left(1+(-1)^{k}\right) \cdot \pi / 2\right),
$$

where $A_{k}$ - set of amplitudes of harmonic series terms;

$\theta_{k}$ - phases of harmonic series terms;

$\tau-$ period of the function (length of the curve);

$x$ - the current length of the curve from the initial to the current point (angle).

To solve the problem of identifying and estimating the deviation parameters, the Digital Image Correlation method [9], digital filters [10] and the enumeration classification method based on Mora polynomials [11] can also be used.

\subsection{Forecasting the assembly geometric parameters of parts}

The first step in creating a predictive model is to create a part assembly in a CAD system that includes 3D parts models that take into account surface shape deviations. Siemens NX system was used in the work. Assembly model creation was carried out in the following 
sequence: fixation of the base part, the disk, by all surfaces; orientation of the mating part,spacer, coaxially (with the disk along the fixing holes) at a specified angle with further contact with the disk; installation of 3D models of bolts concentric to the disk axis; installation of 3D nuts concentric with bolts; setting contact with the spacer. The assembly was saved in ".stp" file.

The next step is to create a finite element model in CAE system. For calculations, the ANSYS Workbench system was used. The created assembly is shown in Figure 1.

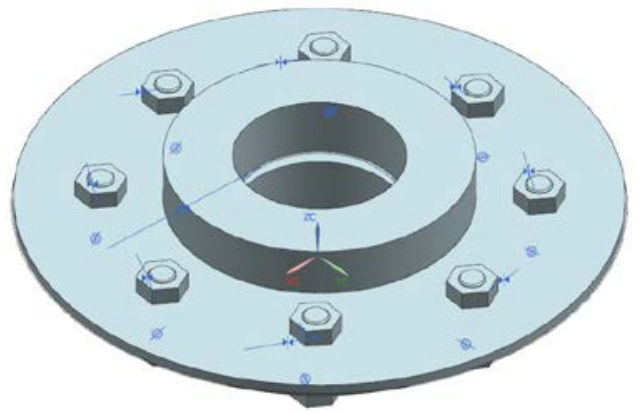

Fig. 1. A computational 3D model that takes into account geometric deviations

Finite elements mesh size is assumed to be equal to $1 \mathrm{~mm}$. An important condition for ensuring calculation reliability is the correct determination of the conditions for contact interaction between surfaces. A "Frictional" type of contact is established between the mating surfaces: the nut end and the spacer simulator; the bolt head end and the disk simulator; the spacer simulator end and the disk simulator. The value of the friction coefficient is 0.15 . The bonded contact is between the mating cylindrical surfaces of the bolt and the nut. The contact stiffness, "Normal Stiffness", between the mating end surfaces of the spacer simulator and the disk simulator having shape deviations was reduced from 1 to 0.2 to ensure the convergence of the calculation.

Since the assembly of parts is carried out consecutively and irregularly, an important condition for ensuring reliability is the account of tightening irregularity of bolt joints. Simulation of eight bolt joints was carried out using the Bolt Pretension function. Tightening simulation is carried out by the "criss-cross" method in 10 stages with the tightening torque $5-50 \mathrm{~N} \cdot \mathrm{m}($ step $5 \mathrm{~N} \cdot \mathrm{m})$. To translate the required wrench torque into the tightening force, we use the following relationships [12]. The torque of the threaded joint was determined based on the frictional force on the end face of the nut by the formula:

$$
M_{T}=\frac{V f_{1}\left(D^{3}-d_{c}^{3}\right)}{3\left(D^{2}-d_{c}^{2}\right)},
$$

where $V$ - tightening force in $\mathrm{H}$;

$d_{c}-$ bolt drilled diameter in $\mathrm{mm}$;

$D$ - nut, bolt head bearing surface diameter in $\mathrm{mm}$.;

$f_{1}$ - coefficient of friction on the bolt bearing surface $f_{1}=0,15$.

The results of calculations are saved in $9 \ldots 81$ stages with the step 8 , for further analysis in ".stp" format. After the calculations are completed, a preliminary analysis of the deformations of the assembly and the elements is carried out as well as pressure values occurring in the contacts. 


\subsection{Algorithm for calculation of assembly parameters}

Evaluation of errors in the assembly of parts is carried out using control surfaces radial and face run-outs. As a result of calculations using CAE systems, a model for assembling parts in STL format is formed. The computation of assembly parameters was carried out in three stages. At the first stage, the surface type was determined by the indicated point of the surface. At the second stage, areas of the surface belonging to the previously defined type were identified. At the third stage, the parameters of surface radial and face run-outs were calculated.

It is known that a part model in STL format consists of a set of triangular sections. To find the face run-out for each point of the model, the distance to the plane specified by a point on the axis of rotation and its directing vector is determined. The specified distance is determined by the formula 3 .

$$
R_{i}=-\frac{(\vec{x}-\vec{p}, \vec{v})}{(\vec{v}, \vec{v})}
$$

where $R_{i}$ - distance from i point of the reference surface to the target plane;

$\vec{x}$ - radius-vector of the point on the axis of rotation of the part;

$\vec{p}_{i}$ - radius-vector of the point of the reference surface;

$\vec{v}$ - directing vector of the axis of rotation.

After all the distances are found, the face run-out is calculated by the formula (4):

$$
D_{F}=\max _{i} R_{i}-\min _{j} R_{j},
$$

где $D_{F}$ - face run-out.

The calculation of radial run-out is carried out in two stages. At the first stage, the reference surface point, the most distant from the axis of rotation, is found. The distance from the point to the axis of rotation is calculated by the formula (5).

$$
R=-\frac{\|\vec{v} \times(\vec{p}-\vec{x})\|}{\|\vec{v}\|},
$$

where $R$ - distance from the point of the reference surface to the axis of rotation of the part;

$\vec{x}$ - radius-vector of the point on the axis of rotation of the part;

$\vec{p}_{i}$ - radius-vector of the point of the reference surface;

$\vec{v}$ - directing vector of the axis of rotation.

At the second stage, the point, the closest to the axis of rotation is found. This point is sought on all the boundaries of the triangular sections of the reference surface. The calculation of radial run-out is performed by the formula (6):

$$
D_{R}=R_{\max }-R_{\min },
$$

To determine the above parameters, RunOutCalculator application was developed. It allows to calculate the parameters based on the 3D model of the part obtained as a result of product measuring with the help of coordinate measuring machines as well as numerical 
simulation of product assembly process performed in CAE systems. A user can set a surface for which the calculation of parameter to be performed and set the axis of rotation.

\section{Results}

In the process of performing theoretical studies, the following parameters were set:

$\neg$ axial force of tightening bolted joints: $10,20,30,40 \mathrm{~N}$;

$\neg$ The amplitude of the deviation of the shape of the surfaces: $0.01 ; 0.02 ; 0.06 ; 0.1 \mathrm{~mm}$.

Figure 2 shows the shape of the deflection of the shape of the end surface of the spacer.

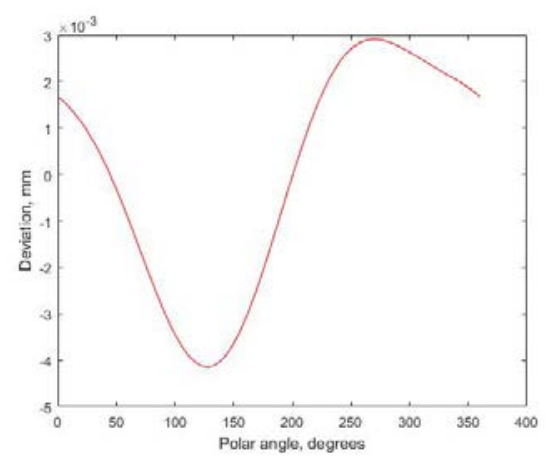

a) Symmetrical deviation

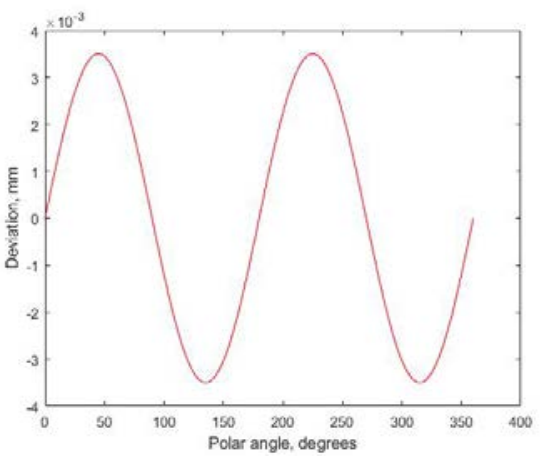

b) Unbalanced deviation

Fig. 2. The deviation of the shape of the end surface of the spacer

Figure 3 shows the dependence of the value of the end run-out on the amplitude of the symmetric deviation of the shape and the axial force of tightening the bolted joint.

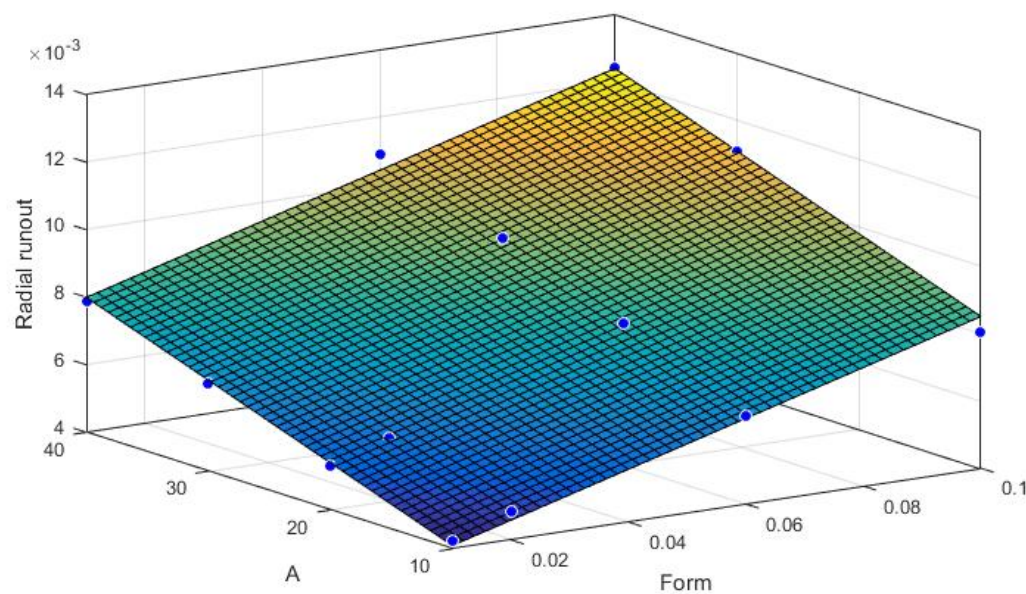

Fig. 3. Dependence of the radial runout on the deviation of the shape and force of the bolted joint

The above results can be described by a linear polynomial dependence:

$$
\Pi_{\mathrm{i}}=\mathrm{a}_{\mathrm{i}}+\mathrm{b}_{\mathrm{i}} * \mathrm{~A}+\mathrm{c}_{\mathrm{i}} * \mathrm{~F},
$$

where $\Pi_{\mathrm{i}}-$ considered assembly parameter, $\mathrm{mm}$; 
ai, bi, ci - polynomial coefficients;

A - amplitude of shape deflection, mm;

$\mathrm{F}$ - axial force of tightening the bolted joint, $\mathrm{N}$.

The results of data approximation are given in Table 1.

Table 1. Parameters of approximating polynomials

\begin{tabular}{|l|l|l|l|l|l|}
\hline Heartbeat & Deviation & $\mathrm{a}_{\mathrm{i}}$ & $\mathrm{b}_{\mathrm{i}}$ & $\mathrm{c}_{\mathrm{i}}$ & $\mathrm{R}^{2}$ \\
\hline Radial & \multirow{2}{*}{ Unbalanced } & 0.002315 & 0.04881 & 0.000129 & 0.986 \\
\cline { 3 - 6 } Run-out & & 0.005423 & 0.9135 & 0.001822 & 0.973 \\
\hline Radial & \multirow{2}{*}{ Symmetrical } & 0.002667 & 0.02377 & 0.000111 & 0.983 \\
\cline { 1 - 4 } Run-out & & 0.002985 & 0.8156 & 0.001537 & 0.998 \\
\hline
\end{tabular}

Close to unity values of the coefficient of determination R2 show the possibility of using the obtained regression dependencies.

\section{Conclusion}

The analysis of the results of the research made it possible to draw the following conclusions:

1. The deviation of the shape of the mating surface of the spacer simulator causes a loose fit and a decrease in the contact stiffness of the surfaces, which leads to an increase in the values of the radial and end beats.

2. Modeling of uneven tightening of threaded joints during assembly allowed to take into account the appearance of distortions and deformations of assembled assembly units.

3. From the simulation results it follows that the predicted assembly parameters have a linear dependence on the magnitude of the deviation of the shape and the axial force of tightening the bolted joint.

The received theoretical results on this technique allow to draw a conclusion about the possibility of its further development with the purpose of industrial introduction to ensure the improvement of the quality of the products to be assembled.

This work was supported by the Ministry of Education and Science of the Russian Federation in the framework of the implementation of the Program State Assignment for 2018. The project code is $9.11560 .2018 / 10.11$.

\section{References}

1. S. Katonaa, M. Kocha, S. Wartzackb, An approach of a knowledge-based process to integrate real geometrymodels in product simulations, 26th CIRP Design Conference Procedia, v. 50, pp.813-818 (2016)

2. X. Yan, A. Ballu, A. Blanchard, S. Mouton and H. Niandou, Development of virtual metrology laboratory based on skin model shape simulation, Advances on Mechanics, Design Engineering and Manufacturing, Lecture Notes in Mechanical Engineering, v. 43, pp. 148 - 153 (2016)

3. J-Y. Dantan, A. Ballu and L. Mathieu, Geometrical product specifications - model for product life cycle, Computer-Aided Design v. 40 (4), p. $493-501$ (2008)

4. S. Samper, P.A. Adragna, H. Favreliere, M. Pillet, Modeling of $2 D$ and $3 D$ assemblies taking into account form errors of plane surfaces, Journal of Computing and Information Science in Engineering, v. 9 (4), pp.041005 (2009) 
5. Z. Zhua, L. Qiaoa, An Improved Tolerance Analysis Method Based on Skin Model Shapes of Planar Parts, 9th International Conference on Digital Enterprise Technology - DET 2016, v. 56, pp.237-242 ( 2016 )

6. J. Guo, B. Li, Z. Liu, J. Hong, X. Wu. Integration of geometric variation and part deformation into variation propagation of 3-D assemblies, International Journal of Production Research, v. 54 (19), pp. 5708-5721 (2016)

7. O.V. Baturin, D.A. Kolmakova, A. Gorshkov. etc., Generation of the equations for the profile losses calculation in blade row of axial turbines in design analysis, v. 2C (2016)

8. A.V. Balaykin, K.A.Bezsonov, M.V. Nekhoroshev, A.P. Shulepov, Developing Parametric Models for the Assembly of Machine Fixtures for Virtual Multiaxial CNC Machining Centers, IOP Conference Series: Materials Science and Engineering, v. 302(1), pp. 012009 (2018)

9. S. R. Abulkhanov, D. L. Skuratov, A. I. Khaymovich, Correlation image analysis of surface roughness, v. 746 (KEM), pp. 296-304 (2017)

10. O.V. Zakharov, A.V. Kochetkov, N.M. Bobrovskij, I.N. Bobrovskij, P.A. Melnikov. Analysis of stationary means of measurement filters with optimum sensitivity. 13th International Scientific-Technical Conference on Actual Problems of Electronic Instrument Engineering, v. 10 (1109) pp. 241-244(2016)

11. V. Musalimov, P. Kovalenk, S. Perepelkina, Estimation of triboresistance during surface nanoscanning 5th World Tribology Congress, WTC 2013,v. 2, pp. 1744-1746 (2013).

12. E.A. Chudakov, S.A. Akopov, I.I. Artobolevsky, N.S. Accherkan, I.M. Besprozvannyi and others, Engineering calculations in machine building (Moscow, State Scientific and Technical Publishing House of Machine-Building Literature, 1947. - 456 from) 